\title{
Superposition of renewal processes for modelling imperfect maintenance
}

\author{
M.J. Kallen \& R.P. Nicolai \\ HKV Consultants, The Netherlands \\ S.S. Farahani \\ Delft University of Technology, The Netherlands
}

\begin{abstract}
In problems of maintenance optimization, it is convenient to assume that repairs are equivalent to replacements and that systems or objects are therefore brought back into an as-good-as-new state after each repair. Standard results in renewal theory may then be applied for determining optimal maintenance policies. In practice, there are many situations in which this assumption can not be made. The quintessential problem with imperfect maintenance is how to model it. In many cases it is very difficult to assess by how much a partial repair will improve the condition of a system or object and it is equally difficult to assess how such a repair influences the rate of deterioration. In this paper, a superposition of renewal processes is used to model the effect of imperfect maintenance. It constitutes a different modelling approach than the more common use of a virtual age process.
\end{abstract}

\section{INTRODUCTION}

The research presented in this paper was motivated by the practice of spot repairing steel coating systems (i.e. paint). As soon as some percentage of the coating is damaged, the damaged spots are repaired by replacing the old coating. Since not all coating is replaced at once, these spot repairs represent partial (or imperfect) repairs of the coating system.

Let us consider the following example: we have a steel structure which is protected against corrosion by a coat of paint. Once 3\% of the total surface of the coating is damaged, these damaged areas are repaired. The remaining $97 \%$ of the surface is left as is. From a modelling point of view the difficulty now lies in the fact that the (random) time to reach the threshold of $3 \%$ damaged surface is different after the repair than it was before. Probabilistically we now have a mixture of two areas with different lifetime expectations. After the second repair, the mixture will become more complicated as we may repair some spots with the old coating, but also some of the spots which received a new layer at the first repair.

Intuitively, the time between repairs will converge to some value as the number of spot repairs increases. This may be easily demonstrated by means of a Monte Carlo simulation as will be done in the examples later on. In the long run the ages of all spots become sufficiently mixed for some form of stationarity to arise. In this paper, the full probability distribution function of the time between repairs will be derived.

Another example of a similar situation is one of a parallel system consisting of a large number of identical components. A certain fraction of the total number of components is allowed to fail before they are collectively replaced. This fraction could be based on the requirement of a minimum system reliability or simply on the fact that the stress on the remaining components will become too large at some point in time.

The basic idea in this paper is the following: the coated surface consists of a grid of cells which represent the spots. If we assume that the arrival in time of damages to the coating in each cell can be modelled by a renewal process, then the arrival of damages on the complete surface is formed by a superposition of these processes. For simplicity we assume that the individual cells are independent. Implicitely we have also assumed that damaged cells are repaired immediately and that the time required for the repair is negligable.

However, in this case, we do not want to repair the cells once they become damaged, but we want to wait until a fraction of the total number of cells is damaged and then repair all of the damaged cells at once. Using the concept of the superposed renewal processes, we will approximate the asymptotic distribution of the time between repairs or, more general, the time to reach a predefined fraction of damaged cells.

Section 2 discusses two approaches to derive the probability distribution function of the interarrival time for a superposed renewal process. Then, Section 3 uses this result to determine the probability distribution function of the time between repairs. These result are demonstrated in Section 4 using a number of examples. The paper ends with a summary and conclusions.

\section{SUPERPOSITION OF RENEWAL PROCESS}

We start by introducing the notation and by giving the necessary definitions. A renewal process $\{N(t), t \geq 0\}$ 
is a non-negative integer-valued stochastic process which registers the successive renewals in the time interval $[0 ; t]$. Let each renewal process have interarrival times $X_{1}, X_{2}, \ldots$ and let $S_{k}=\sum_{i=1}^{k} X_{i}, k \geq 1$, be the time of the $k$-th renewal. Each interarrival time is identically and independently distributed according to some non-negative probability distribution function $F(x)=\operatorname{Pr}\left\{X_{i} \leq x\right\}, x \geq 0$. The hazard rate is given by the familiar expression $v(x)=f(x) / R(x)$, where $R(x)=1-F(x)$ is the survival probability at a time $x$ since the last renewal. The following well known relationships will also be required later on:

$$
R(x)=\exp \left\{-\int_{u=0}^{x} v(u) d u\right\}
$$

and

$f(x)=v(x) \exp \left\{-\int_{u=0}^{x} v(u) d u\right\}$.

The rate of occurence (or the average process rate) is $1 / \mu$, where

$\mu=\int_{x=0}^{\infty} x d F(x)$

is the mean time between renewals. For more on the theory of renewal processes, we refer to any textbook on stochastic processes like, for example, Ross (2000).

A superposed renewal process, here denoted by $N_{s}(t)$, is obtained by counting the renewals up to time $t$ of multiple source processes, each of which is a renewal process:

$N_{S}(t)=\sum_{i=1}^{n} N_{i}(t), \quad n \geq 1$.

Unless the individual renewal processes $N_{i}(t)$ are Poisson processes, the superposed process itself is not a renewal process. This is because the interarrival times are no longer independent. However, the distribution of the interarrival time of the superposed process can be derived analytically. Moreover, it is well known that the superposed process is close to being a Poisson process when the number of sources is very large.

In the following, we will discuss two distinctively different approaches to the derivation of the distribution of the interarrival time of the superposed process. One is the approach by Torab and Kamen (2001) using a rate-optimal approximation of the superposed process and the other is the approach by Cox and Smith (1954) using what is known as the equilibrium distribution of a renewal process. Given the same assumptions about the individual renewal processes, we will show that these two approaches yield the same result. In the next section, we will show how this result can be used to obtain an approximation of the interrepair time distribution. Any (probability distribution) function associated with the superposed process will be denoted with a subscript $\mathrm{s}$. We begin with the approach used by Cox and Smith (1954).

\subsection{Cox and Smith (1954)}

First, we give a brief account of the main contribution in the paper. Let $Y(t)$ denote the age of the process at time $t: Y(t)=t-S_{N(t)}$, with $S_{N(t)}$ the time of the last renewal before $t$. Cox and Smith (1954) referred to this as the delay time. If the interarrival time distribution $F(x)$ is not lattice, then

$\lim _{t \rightarrow \infty} \operatorname{Pr}\{Y(t) \leq x\}=\frac{1}{\mu} \int_{u=0}^{x} R(u) d u$.

This limit distribution is referred to as the equilibrium distribution for $F$, see Ross (1970, Chapter 3), and we will denote it by $G(x)$. For a superposed process, $Y_{s}(t)$ is equal to the smallest age of the individual source processes: $Y_{s}(t)=\min \left\{Y_{1}(t), \ldots, Y_{n}(t)\right\}$. The distribution can thus be easily derived as follows

$\operatorname{Pr}\{Y(t)>x\}=\prod_{i=1}^{n} \operatorname{Pr}\left\{Y_{i}(t)>x\right\}=[1-G(x)]^{n}$,

where we assume that $t$ is large or, in other words, that the process has been running for a long time. The density function of Eq. (5) is

$g_{s}(x)=n \frac{R(x)}{\mu}[1-G(x)]^{n-1}$,

as Eq. (4) implies $g(x)=R(x) / \mu$. Now Cox and Smith (1954) observed that the interarrival density of a renewal process, $f(x)$, may be obtained from the equilibrium density of the process, $g(x)$, by taking the derivative of $g(x):(d g / d x)=-f(x) / \mu$. Differentiating Eq. (6), Cox and Smith (1954) thus arrived at the following result for the density function of the interarrival time distribution for the superposed process:

$f_{S}(x)=-\frac{d}{d x}\left\{R(x)[1-G(x)]^{n-1}\right\}$.

This result may be further reduced to

$f_{s}(x)=R(x)[1-G(x)]^{n-1}\left[v(x)+(n-1) \frac{R(x)}{\Psi(x)}\right]$,

where

$\Psi(x)=\int_{u=x}^{\infty} R(u) d u$

is a notation used by Torab and Kamen (2001). Note that $\Psi(0)=\mu$ and that $\Psi(x) / \mu=1-G(x)$.

In Eq. (5), it was implicitely assumed that the interarrival distributions of the source processes are identical. In the next section, we describe the approach used by Torab and Kamen (2001) which does not require this restriction. However, we will show that both approaches give the same result if we do make this assumption. 


\subsection{Torab and Kamen (2001)}

In a rather enjoyable paper, Torab and Kamen (2001) approach the characterization of the superposed process in terms of the process rates. Working with renewal rates avoids the difficulties involved when working with distributions of the interarrival time, especially when this distribution is not exponential. The rate of the renewals in a superposed process, for example, is simply equal to the sum of the rates of the source processes.

Knowing that the superposed process is not a renewal process, Torab and Kamen (2001) construct a renewal process with a rate function $v_{s}(x)$ which minimizes the difference with the actual superposed process. In other words: they construct a renewal process which is a rate-optimal approximation of the superposed process. They find that the optimal rate function is the following:

$v_{s}(x)=\frac{\sum_{i=1}^{n} \eta_{i}(x) \cdot\left\{v_{i}(x)+\sum_{j=1, j \neq i}^{n} \eta_{j}(x)\right\}}{\sum_{i=1}^{n} \eta_{i}(x)}$,

where

$\eta(x)=R(x) / \Psi(x)$

is named the recurrence rate. $v_{i}(x)$ and $\eta_{i}(x)$ are resp. the hazard rate and the recurrence rate of the source process $i$ with $i=1, \ldots n$. This rate is obtained by taking $\eta(x)=g(x) /[1-G(x)]$ (see Eq. 3) so it may be interpreted as being the rate of the equilibrium distribution $G(x)$. Also, it can be shown, using Eq. (1), that

$\eta(x)=-\frac{d}{d x} \ln \left[\frac{\Psi(x)}{\mu}\right]$.

This result follows by setting $1-G(x)=\Psi(x) / \mu=$ $\exp \left\{-\int_{u=0}^{x} \eta(u) d u\right\}$ and solving for $\eta(x)$.

If we now assume that all source processes have the same interarrival distribution $F(x)$, then $v_{i}(x)=v(x)$ and $\eta_{i}(x) \equiv \eta(x)$ for all $i$ and Eq. (9) reduces to

$v_{s}(x)=v(x)+(n-1) \eta(x)$.

We can now use Eq. (2), together with the relationship in Eq. (11), to derive the interarrival distribution $f_{s}(t)$ of the (approximated) superposed process. First, we determine the integral of $v_{s}(x)$ :

$$
\begin{aligned}
& \int_{u=0}^{x} v_{s}(u) d u= \\
& =\int_{u=0}^{x} v(u) d u+(n-1) \int_{u=0}^{x}-\frac{d}{d u} \ln \left\{\frac{\Psi(u)}{\mu}\right\} d u \\
& =-\ln R(x)-(n-1)\left[\ln \left\{\frac{\Psi(x)}{\mu}\right\}-\ln \left\{\frac{\Psi(0)}{\mu}\right\}\right] \\
& =-\ln \left(R(x)\left\{\frac{\Psi(x)}{\mu}\right\}^{n-1}\right) .
\end{aligned}
$$

Next, the density function $f_{s}(x)$ of the interarrival time is found by substituting Eqs. (12) and (13) into Eq. (2). This gives the same result as in Eq. (8).

\subsection{Large number of sources}

It may be easily checked that if $f(x)=\exp \{-x / \mu\}$, i.e. the source processes are Poisson processes with rate $1 / \mu$, then the superposed process is also a Poisson process with rate $n / \mu$ with $n \geq 1$ being the number of sources.

There is also a number of ways to show that the superposed process is close to a Poisson process if the number of sources is sufficiently large. This means that the interarrival distribution tends towards the exponential distribution as $n$ grows large. For example, let the time between failures be a multiple of $\mu / n: x=\tau \mu / n$. Then $x \rightarrow 0$ when $n \rightarrow \infty$ and $v_{s}(x) \rightarrow n / \mu$ in Eq. (12). Cox and Smith (1954) use a similar approach to show that the number of events during a period $x$ tends to be Poisson distributed with rate $1 / \tau$. A good overview of the main proofs of this result is given by (Barlow and Proschan, 1965, Ch. 2, Sec. 3).

\section{INTER-REPAIR TIME DISTRIBUTION}

Let a repair be performed when the coating in $k=\rho n$ cells is damaged. Here $\rho$, with $0<\rho<1$, is a fraction which is defined such that $k=1,2, \ldots$ If the number of source processes $n$ is sufficiently large, we may approximate the superposed process with a Poisson process with rate $n / \mu$, where $\mu$ is the mean time between failures in the source processes. However, once a cell becomes damaged, the source process actually stops and the rate of the superposed process is reduced by $1 / \mu$. This means that the superposed process is a continuous-time Markov process which has a rate in state $i$ equal to

$v_{i}=\frac{n}{\mu}\left(1-\frac{i}{n}\right), \quad 0 \leq i \leq n$.

Here, $i$ corresponds to the number of failed cells. The time required to reach $k$ failed cells is now given by the time to reach state $k$ when starting in state 0 . This is known as a phase-type distribution (Neuts, 1981), but the inter-repair time distribution in this particular case is also known as a hypoexponential distribution:

$f_{k}(x)=\sum_{i=0}^{k-1}\left[\prod_{j \neq i} \frac{v_{i}}{v_{i}-v_{j}}\right] \frac{1}{v_{i}} \exp \left\{-\frac{x}{v_{i}}\right\}$,

with $v_{i} \neq v_{j}$ for $i \neq j$. See, for example, Ross (2000, p. 253) or Johnson et al. (1994, p. 552) where it is referred to as the general gamma or general Erlang distribution.

\section{EXAMPLES}

\subsection{Monte Carlo simulation}

In order to check the approximation of the interarrival time distribution, we perform a Monte Carlo simulation. Comparing the result of the approximation with 
that of the simulation gives us an indication of how the approximation fairs in different cases. The simulation is performed as follows. First we choose a sufficiently large number of source processes $n$ and number of repairs $m$. Then we start by taking $n$ samples from the interarrival time distribution $F(x): x_{1}, x_{2}, \ldots, x_{n}$. Next, we repeat the following steps $m$ times:

1. sort the samples in ascending order such that

$$
x_{(1)} \leq x_{(2)} \leq \cdots \leq x_{(n)}
$$

and store the time of $x(k)$;

2. replace times $x_{(1)}$ to $x_{(k)}$ with new samples of the interarrival time (sampling again from $F(x)$ ) and add the time $x_{(k)}$ to these new samples.

At the end, we will have $\mathrm{m}$ simulated inter-repair times by taking the time between the stored values of $x_{(k)}$. In the following two sections we will show the results for $F(x)$ being a exponential and Weibull distribution. The result for the exponential distribution is known analytically, which enables us to check the correctness of the simulation algorithm presented here.

\subsection{Exponential distribution}

Let the interarrival distribution of each source process be the exponential distribution

$F(x)=1-\exp \{-x / \mu\}, \quad x \geq 0$,

with mean interarrival time $\mu=5$. We set $\rho=0.03$, which means that we repair the damaged cells once $3 \%$ of the total number of cells have deteriorated. For the simulation we use $n=500$ and $m=100$, such that $k=15$. The result for the inter-repair time distribution, using the superposed process, is given in Fig. 1. As expected, the inter-repair time obtained using Eq. (15) agrees with the simulation results. This agreement indicates that the simulation algorithm presented in the previous section is correct.

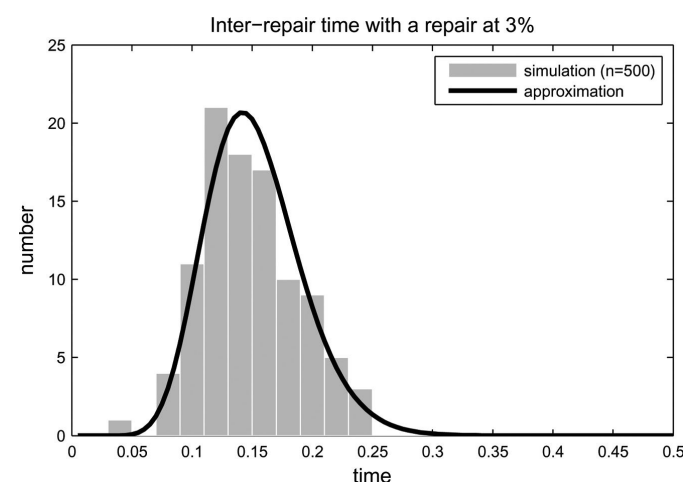

Figure 1. Inter-repair time distribution for a superposed process consisting of 500 source processes with exponential interarrival times. Damaged cells are repaired once 3\% of the total surface area is damaged.

\subsection{Weibull distribution}

Let the interarrival distribution of each source process be the Weibull distribution

$F(x)=1-\exp \left\{-\left[\frac{x}{a}\right]^{b}\right\}, \quad x \geq 0$,

with scale parameter $a=5$ and shape parameter $b=2.5$. The mean interarrival time $\mu$ for each process is approximately 4.44 . Figure 2 shows how the time between repairs initially decreases as the number of repairs increases, after which it varies around a mean value of approximately 0.13 . This is the mean value of the inter-repair time distribution obtained using Eq. (15). Figure 3 compares this distribution with a histogram of the simulated times between repairs. We know this result is not exact, but the approximation is good.

The quality of the approximation depends on a number of aspects. First of all, the number of source processes $(n)$ must be sufficiently large. As individual cells become damaged, the total number of source

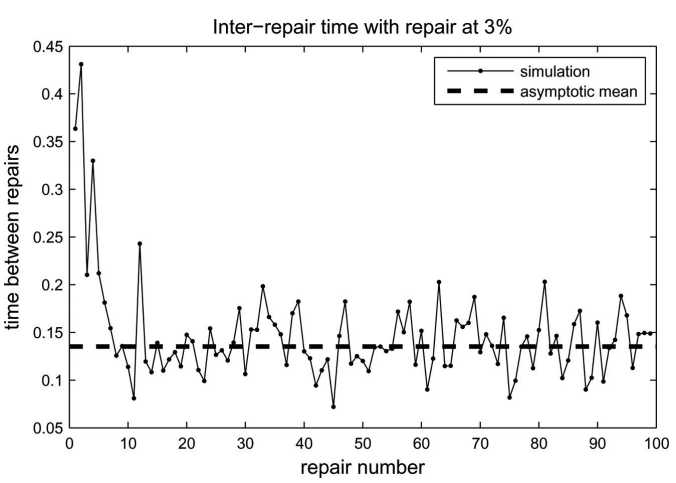

Figure 2. Inter-repair time between each repair for a superposed process consisting of 500 source processes with weibull distributed interarrival times. Damaged cells are repaired once $3 \%$ of the total surface area is damaged.

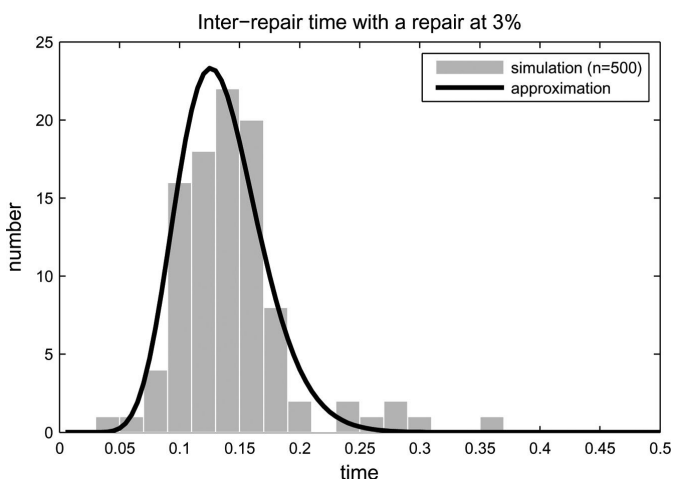

Figure 3. Inter-repair time distribution for a superposed process consisting of 500 source processes with weibull distributed interarrival times. Damaged cells are repaired once $3 \%$ of the total surface area is damaged. 


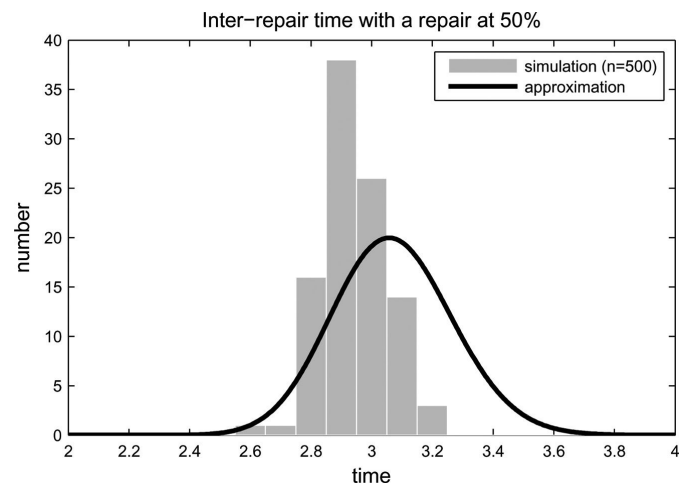

Figure 4. Inter-repair time distribution for a superposed process consisting of 500 source processes with weibull distributed interarrival times. Damaged cells are repaired once $50 \%$ of the total surface area is damaged.

processes becomes smaller. Therefore, the fraction of damaged surface area also affects the quality of the approximation adversely: the larger the fraction $\rho$, the worse the approximation. This is demonstrated in Fig. 4, which is the same example with $\rho=$ 0.50 . In this case, the approximated inter-repair time distribution overestimates the true inter-repair time (slightly).

Finally, the quality of the approximation will be poorer for an interarrival time distribution $F(x)$ which is less "exponential". For the Weibull distribution this means that the quality of the approximation will decrease as the shape parameter $b$ deviates more from 1 . The approximation in Fig. 4 with a repair at $50 \%$ damaged area will become better if the shape parameter is closer to 1 .

To summarize: as the number of source processes becomes smaller, the mean time between failures becomes larger; and if the mean time between failures becomes larger, the influence of the failure rate of the interarrival time distribution $f_{s}(x)$ becomes larger. Figure 5 shows three different failure rate functions $v_{s}(x)$ for $f_{s}(x)$ : one based on the assumption that the superposed process is Poisson $(=n / \mu$, which is used in these examples), one based on the rate-optimal approximation $\left(=v_{s}(x)\right.$ as in Eq. 12) and one obtained by simply summing the rate functions of the source processes $(=n v(x))$. The latter is clearly not a good choice for $v_{s}(x)$. Also, the fact that $v_{s}(x) \rightarrow n / \mu$ as $x \rightarrow 0$ (see Sec. 2.3) is confirmed here.

Note that the rate defined in Eq. (12) is slightly greater than the rate $n / \mu$ which we used here. Looking at Fig. 4, we conclude that the approximation overestimates the true time between repairs. The approximated superposition process with failure rate $n / \mu$ is too slow. By this we mean that, in reality, the failure of cells occurs at a slightly higher rate. From this, we conclude that the approximation will improve if we use the rate in Eq. (12), which was proposed by Torab and Kamen (2001). However, since this rate is not constant, we will not be able to use the hypoexponential distribution in Eq. (15). This is because the

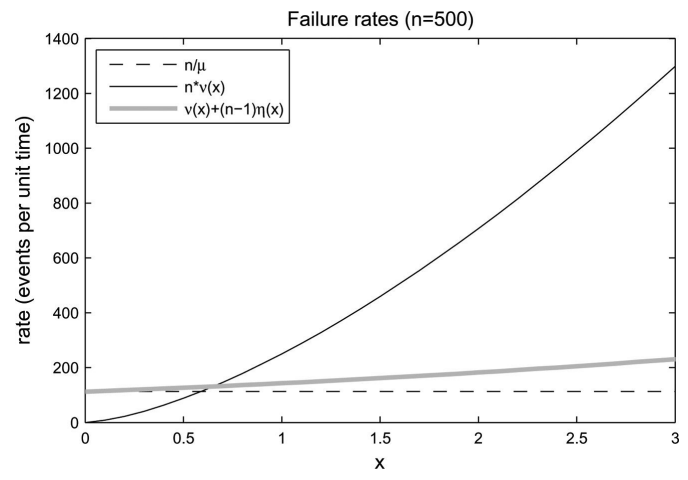

Figure 5. Comparison of failure rate functions for a superposed process consisting of 500 source processes with weibull distributed interarrival times.

superposed process will no longer be continuous-time Markov process, but rather a semi-Markov process. The phase-type distribution for the time to reach a certain number of failed cells may also be determined for a semi-Markov process, but this is beyond the scope of this paper.

\section{CONCLUSIONS}

In this paper we proposed a model for partial repair (or imperfect maintenance) based on the superposition of renewal processes. We showed how this model may be used to determine an approximate (asymptotic) probability distribution of the time between repairs. Failed cells or components are not repaired until a predefined fraction of the total number has failed.

The quality of the approximation of the inter-repair time distribution is good, but deteriorates once the number of source processes decreases. This is especially the case when the interarrival time of the source processes is non-exponential. Using Taylor expansions, Blumenthal et al. (1973) derived correction factors for the exponential interarrival time if the number of source processes $n$ is small or $t$ is finite. A finite time $t$ introduces an error due to the fact that the process has not been running very long, which means that the asymptotic results may not be valid. Whether or not these correction factors can be used as an indication of the minimum number of source processes is the subject of further research.

In extreme cases, such as when the number of source processes is small or when many failures are allowed to occur before a repair is made, the superposed process will have to be modelled by a semi-Markov process based on the rate-optimal approximation of Torab and Kamen (2001).

There are many applications for this model. We have mentioned two: spot-repair of a protective coating system used for objects made of steel and the replacement of failed components in a large parallel system. The example of the steel coating is an idealized example, 
since the assumption of independent spots will not be true in practice. Also, protective coating systems may not be replaced infinitely many times (as the steel is damaged during the process of removing the damaged coating) and spots adjacent to damaged spots will generally also be repainted. Nonetheless, the approximation proposed in this paper gives the modeller a useful tool for riskbased maintenance optimization. The model allows for a quantification of the overall rate of deterioration, and the uncertainty about this rate, for a partially repaired system.

\section{ACKNOWLEDGMENTS}

The authors would like to thank Hans van der Weide at the Delft University of Technology for his helpful comments during the research for this paper. We also thank an anonymous reviewer for pointing out the fact that the recurrence rate $\eta(x)$ is the reciprocal of the mean residual lifetime:

$E[T-x \mid T>x]=\frac{1}{R(x)} \int_{u=x}^{\infty} R(u) d u=\frac{\Psi(x)}{R(x)}$,

where $T$ is the time between failures.
This paper is dedicated to the memory of prof. Jan M. van Noortwijk ( $\star 1961-\dagger 2008)$ who was the supervisor of Ms. Farahani during her research project.

\section{REFERENCES}

Barlow, R.E. \& Proschan, F., 1965. Mathematical Theory of Reliability. John Wiley \& Sons, New York, NY.

Blumenthal, S., Greenwood, J.A., \& Herbach, L., 1973. The transient reliability of series systems of superimposed renewal processes. Technometrics, 15(2):255-269.

Cox, D.R. \& Smith, W.L., 1954. On the superposition of renewal processes. Biometrika, 41:91-99.

Johnson, N.L., Kotz, S., \& Balakrishnan, N., 1994. Continuous univariate distributions, Volume 1. John Wiley \& Sons, 2nd edition.

Neuts, M.F., 1981. Matrix-geometric solutions in stochastic models: an algorithmic approach. John Hopkins University Press, Baltimore, MD.

Ross, S.M., 1970. Applied Probability Models with Optimization Applications. Holden-Day, San Francisco, CA.

Ross, S.M., 2000. Introduction to Probability Models. Harcourt/Academic Press, Burlington, MA, 7th edition.

Torab, P. \& Kamen, E.W., 2001. On approximate renewal models for the superposition of renewal processes. In IEEE International Conference on Communications (ICC 2001), Helsinki, Finland, June 11-14, 2001, volume 9, pages 2901-2906. 